\title{
The future of pharmacogenetics in the treatment of hypertension
}

\author{
Patrick N Cunningham*,1 \& Arlene B Chapman ${ }^{1}$ \\ ${ }^{1}$ Section of Nephrology, University of Chicago, IL 60637, USA \\ *Author for correspondence: pcunning@medicine.bsd.uchicago.edu
“'a streamlined pharmacogenomic approach to get hypertensive patients on the most effective, efficient and well-tolerated drug regimen would be enormously worthwhile."

First draft submitted: 30 November 2018; Accepted for publication: 30 November 2018; Published online: 27 February 2019

\begin{abstract}
Keywords: beta blocker • blood pressure • calcium channel blocker • pharmacogenomics • single nucleotide polymorphism • thiazide
\end{abstract}

Hypertension (HTN) is one of the most common, serious health conditions worldwide, and is responsible for significant morbidity and mortality, through its contribution to vascular disease, congestive heart failure, cerebrovascular disease and kidney failure. In USA, it is estimated to affect almost 80 million Americans. Despite the availability of multiple drugs to treat HTN, rates of control of blood pressure (BP) are less than $50 \%$, and interindividual variability of BP response to different agents is high. While certain demographic factors such as age, gender and race may help guide the selection of one antihypertensive agent over another, to a large extent the strategy that patients receive is based on trial and error.

The sequencing of the human genome in 2003 was a major medical breakthrough and ushered in hopes for an individualized HTN treatment approach for patients. Not only could genetic information be used to diagnose or understand the pathophysiology of disease, but the field of pharmacogenomics brought the promise of tailoring individual drugs and their dosage to each individual's genetic background. The Clinical Pharmacogenomics Implementation Consortium (CPIC) is an organization which reviews the large and growing pharmacogenomics published datasets to develop specific recommendations for clinical application [1]. Currently, CPIC lists specific recommendations for 35 drugs, including warfarin, clopidogrel, codeine and amitriptyline among others, in whom genetic testing can guide dosing strategy and selection of specific agents, with the hope that this strategy improves effectiveness and/or decreases adverse events. However, current CPIC guidelines include limited information and no firm recommendations about genetic testing for any antihypertensive drug. Genetic variation in the CYP2D6 gene impacts metoprolol metabolism, and the Dutch Pharmacogenomic Working Group has recommended CYP2D6 screening be used when metoprolol is prescribed, marking the only published pharmacogenomic guideline pertaining to antihypertensives [2].

Despite a large and growing number of published studies, pharmacogenetics research has found that multiple genetic variants have a modest influence on individual response to different antihypertensive medications, consistent with hypertension being a complex medical condition where hundreds of genes influence BP response in a small way [3]. The field of HTN pharmacogenomics has generated some conflicting and inconsistent findings with regard to $\mathrm{BP}$ response to antihypertensive medication, so it is important to define the biologic rationale behind the genetic associations found. A concise summary of genetic variants affecting antihypertensive response is given in Table 1 . $N E D D 4 L$ affects the expression of renal distal tubular sodium channels such as $\mathrm{ENaC}$ through ubiquitination. The $\mathrm{G}$ allele at rs414960 is associated with salt-sensitive hypertension, lower plasma renin levels and greater BP response to thiazide diuretics in analysis of three independent clinical trial cohorts: NORDIL, PEAR and INVEST. The NORDIL and INVEST studies found fewer cardiovascular events in hypertensive carriers of the G allele in those treated with thiazide diuretics [4]. The gene ADRB1 has two independent SNPs, rs1801252 and rs1801253, which lead to amino acid substitutions affecting $\beta$-1 receptor function, BP response to beta-blockers and differential effects on patient survival when atenolol was compared with verapamil therapy (hazard ratio of 2.31) [5].

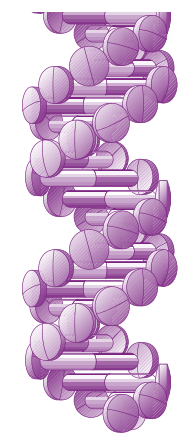

\section{.}

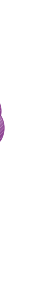


Table 1. Key genetic variants associated with blood pressure response.

\begin{tabular}{|c|c|c|c|c|c|}
\hline Drug & Gene & Variant(s) & Clinical findings & Studies & Ref \\
\hline \multirow[t]{4}{*}{ Thiazides } & NEDD4L & rs4149601 & $\begin{array}{l}\mathrm{G} \text { allele associated with greater BP response, better outcomes with } \\
\text { diuretics }\end{array}$ & PEAR1, INVEST, NORDIL & {$[4]$} \\
\hline & YEATS4 & rs7297610 & $\begin{array}{l}\text { CC genotype greater BP response in African-Americans, with } \\
\text { greater leukocyte expression of YEATS4 and greater decline after } \\
\text { HCTZ treatment }\end{array}$ & GERA1, PEAR1 & [6] \\
\hline & PKCA & rs16960228 & A allele carriers have greater BP response than $\mathrm{GG}$ genotype & $\begin{array}{l}\text { GERA1, PEAR1, GENRES, } \\
\text { NORDIL }\end{array}$ & [7] \\
\hline & GNAS-EDN3 & rs2273359 & $\mathrm{G}$ allele carriers have greater $\mathrm{BP}$ response than $\mathrm{CC}$ genotype & GERA1, PEAR1, NORDIL & [7] \\
\hline \multirow[t]{4}{*}{ Beta-blockers } & ADRB1 & $\begin{array}{l}\text { rs } 1801252 \\
\text { rs1801253 }\end{array}$ & $\begin{array}{l}\text { A allele ( } 49 \mathrm{Gly} \text { ) has greater DBP response } \\
\mathrm{C} \text { allele ( } 389 \mathrm{Gly}) \text { has greater DBP response } \\
49 \text { Ser-389Arg haplotype has mortality benefit in atenolol versus } \\
\text { verapamil }\end{array}$ & INVEST & {$[5$} \\
\hline & GRK4 & $\begin{array}{l}\text { rs } 1024323 \\
\text { rs } 2960306 \\
\text { rs } 1801058\end{array}$ & $\begin{array}{l}\text { 65Leu-142Val haplotype have worse BP response to beta blockers } \\
\text { A allele (486Val) increases cardiovascular events }\end{array}$ & $\begin{array}{l}\text { AASK, PEAR1 } \\
\text { INVEST }\end{array}$ & {$[8$} \\
\hline & PTPRD & $\begin{array}{l}\text { rs12346562 } \\
\text { rs10739150 }\end{array}$ & $\begin{array}{l}\text { A allele predicts SBP response to atenolol in Caucasian population } \\
G \text { allele predicts SBP response to atenolol in African-Americans }\end{array}$ & PEAR1, GENRES & {$[9$} \\
\hline & $\begin{array}{l}\text { SLC25A31 } \\
\text { LRRC15 }\end{array}$ & $\begin{array}{l}\text { rs201279313 } \\
\text { rs11313667 }\end{array}$ & $\begin{array}{l}\text { Deletion allele predicts to greater DBP response in } \\
\text { African-Americans } \\
\text { Deletion allele predicts to greater SBP response in } \\
\text { African-Americans }\end{array}$ & PEAR1, PEAR2 & {$[10$} \\
\hline Metoprolol & CYP2D6 & $* 3, * 4$, others & Poor metabolizers more sensitive, at risk for bradycardia & & {$[2$} \\
\hline Verapamil & KCNMB1 & $\begin{array}{l}\text { rs11739136 } \\
\text { rs2301149 }\end{array}$ & $\begin{array}{l}65 \text { Lys achieves faster BP control with verapamil } \\
110 \text { Leu protects against cardiovascular outcomes in verapamil } \\
\text { group }\end{array}$ & INVEST & {$[11]$} \\
\hline Losartan & NPHS1 & rs3814995 & A allele (117Lys) has greater SBP and DBP response & GENRES, GERA2, SOPHIA & [12 \\
\hline
\end{tabular}

Genetic variation contributing to drug responses identified by the unbiased Genome Wide Association Study (GWAS) approach is held to a high level of statistical significance and validation in an independent cohort. Previously unknown genetic influences such as rs7297610, near YEATS4, were found to be associated with increased diastolic BP response to thiazide diuretics within African-Americans in the GERA trial, a result that was subsequently validated in the PEAR1 trial. The protein product of YEATS4 is a transcription factor whose expression levels are significantly higher but then decrease following thiazide treatment in those carrying the $\mathrm{C}$ as opposed to the $\mathrm{G}$ allele, lending biological plausibility to its role [6]. Most individual HTN pharmacogenomics trials have been small in size, so the combination of several trials for meta-analyses through consortia such as ICAPS (International Consortium for Antihypertensives Pharmacogenomics Studies) is an active approach used to improve statistical power. ICAPS now has 29 clinical trials with 345,000 participants' data available for analysis. The missense variant rs3814995 in NPHS1 (Nephrin), which was initially identified in the relatively small Finnish GENRES trial as associated with an increased BP response to losartan monotherapy, was then validated through collaboration with ICAPS in Caucasian participants from the GERA2 and SOPHIA cohorts [12].

Several limitations in the study of HTN pharmacogenomics remain. BP measurements are intrinsically a 'noisy' measurement to study, with high minute to minute variability, affected by diet, activity, posture, psychological stress and a variety of other factors. In addition, the method of BP measurement also contributes to the noise. Both PEAR and GERA trials made a concerted effort to address this issue by using automated home BP readings and ambulatory BP measures. All GWAS studies, including GWAS studies in the HTN pharmacogenomics field, exclude rare genetic variants with allele frequencies $<0.05$ which could conceivably have large effects on BP response. Whole genome sequencing will address this limitation and may eventually become more widespread to allow for clinical information about these uncommon alleles. Most of the information to date has been obtained from Caucasian populations, which may not necessarily be validated in other racial groups [13]. This is important given that African-Americans have a greater prevalence and severity of HTN, known differences in BP regulatory physiology and greater HTN-related end organ damage. Future work will need to generate more information about non-Caucasian populations to broaden the impact of pharmacogenomics. The generalizability of findings may also be limited by the necessary exclusion of sicker patients with more complex comorbidities in these trials, or 
patients with drug-resistant HTN. Perhaps most important, the number of genetic variants that have been found to influence BP response to antihypertensive agents is large and growing, yet each has a relatively small effect size. As an approach to address this, some preliminary studies have combined the influence of multiple alleles as an overall risk score influencing outcomes [14]. Ultimately, computational algorithms to integrate this data from multiple genes to produce optimum guidance for each patient will move implementation of pharmacogenomics into the clinic.

Valuable genetic information is being discovered related to BP response to antihypertensive agents. However, translating these discoveries into clinical practice to improve treatment of HTN remains a challenge. While pharmacogenetics testing has made inroads in oncology, cancer is a situation where drug efficacy may have immediate impact on short-term survival and drug toxicity may be extremely severe. Primary care physicians treating the majority of HTN are likely to have considerable inertia and persist with the trial and error approach to BP management, given the multiple drug options available. Many current physicians had their medical training in an era before the sequencing of the human genome was achievable, and may vary widely in their comfort level interpreting and using pharmacogenomic data to make treatment decisions for their patients. It may be more realistic for clinical HTN specialists at academic medical centers to be the first group of physicians to implement pharmacogenomics information delivered by a clinical decision support system. Several academic medical centers are already piloting programs that supply a limited panel of genetic testing and recommendations to providers [15,16]. Patients with difficult to control HTN are a subset in whom this approach may be helpful, as well as being a group who may have the most to gain. Another obvious obstacle is the cost of genetic testing. While advancing technology has made genetic testing less expensive, obtaining genetic information (only required once) on an individual patient will ultimately require insurance coverage. As is true of the early phase of many new technologies, medical practice is thus currently in a state where the availability of this technology awaits a demand from practitioners, yet practitioner demand will remain low until the availability of genetic data becomes more widespread and mainstream.

Despite these difficulties, a streamlined pharmacogenomic approach to get hypertensive patients on the most effective, efficient and well-tolerated drug regimen would be enormously worthwhile. This would result in fewer patient visits to readjust BP medications, fewer drugs per patient and potentially better compliance with their medical regimen. Better BP control would lead to fewer cardiovascular and renal complications, and improvement in quality of life and longevity in our hypertensive patients. Given the vast numbers of patients with HTN, this could as well result in enormous cost savings, with one recent economic analysis estimating national cost savings of $\$ 42$ billion over a 3-year period for 10 million insured patients [17]. Demonstration of such improved outcomes in clinical trials would be a powerful stimulus to bring pharmacogenomics into clinical use in patients with HTN and fulfill the promise of personalized medicine.

Financial \& competing interests disclosure

The authors have no relevant affiliations or financial involvement with any organization or entity with a financial interest in or financial conflict with the subject matter or materials discussed in the manuscript. This includes employment, consultancies, honoraria, stock ownership or options, expert testimony, grants or patents received or pending, or royalties.

No writing assistance was utilized in the production of this manuscript.

\section{References}

1. Caudle KE, Klein TE, Hoffman JM et al. Incorporation of pharmacogenomics into routine clinical practice: the Clinical Pharmacogenetics Implementation Consortium (CPIC) guideline development process. Curr. Drug Metab. 15, 209-217 (2014).

2. Swen JJ, Nijenhuis M, de Boer A et al. Pharmacogenetics: from bench to byte - an update of guidelines. Clin. Pharmacol. Ther. 89, 662-673 (2011).

3. Evangelou E, Warren HR, Mosen-Ansorena D et al. Genetic analysis of over 1 million people identifies 535 new loci associated with blood pressure traits. Nat. Genet. 50, 1412-1425 (2018).

4. McDonough CW, Burbage SE, Duarte JD et al. Association of variants in NEDD4L with blood pressure response and adverse cardiovascular outcomes in hypertensive patients treated with thiazide diuretics. J. Hypertens. 31, 698-704 (2013).

5. Pacanowski MA, Gong Y, Cooper-Dehoff RM et al. Beta-adrenergic receptor gene polymorphisms and beta-blocker treatment outcomes in hypertension. Clin. Pharmacol. Ther.84, 715-721 (2008).

6. Duarte JD, Turner ST, Tran B et al. Association of chromosome 12 locus with antihypertensive response to hydrochlorothiazide may involve differential YEATS4 expression. Pharmacogenomics J. 13, 257-263 (2013). 
7. Turner ST, Boerwinkle E, O'Connell JR et al. Genomic association analysis of common variants influencing antihypertensive response to hydrochlorothiazide. Hypertension 62, 391-397 (2013).

8. Vandell AG, Lobmeyer MT, Gawronski BE et al. G protein receptor kinase 4 polymorphisms: beta-blocker pharmacogenetics and treatment-related outcomes in hypertension. Hypertension 60, 957-964 (2012).

9. Gong Y, McDonough CW, Beitelshees AL et al. PTPRD gene associated with blood pressure response to atenolol and resistant hypertension. J. Hypertens. 33, 2278-2285 (2015).

10. Gong Y, Wang Z, Beitelshees AL et al. Pharmacogenomic genome-wide meta-analysis of blood pressure response to beta-blockers in hypertensive African Americans. Hypertension 67, 556-563 (2016).

11. Beitelshees AL, Gong Y, Wang D et al. KCNMB1 genotype influences response to verapamil SR and adverse outcomes in the INternational VErapamil SR/Trandolapril STudy (INVEST). Pharmacogenet. Genomics 17, 719-729 (2007).

12. Hiltunen TP, Donner KM, Sarin AP et al. Pharmacogenomics of hypertension: a genome-wide, placebo-controlled cross-over study, using four classes of antihypertensive drugs. J. Am. Heart Assoc. 4, e001521 (2015).

13. Gong $\mathrm{Y}, \mathrm{McD}$ Donough $\mathrm{CW}$, Wang $\mathrm{Z}$ et al. Hypertension susceptibility loci and blood pressure response to antihypertensives: results from the pharmacogenomic evaluation of antihypertensive responses study. Cir. Cardiovasc. Genet. 5, 686-691 (2012).

14. McDonough CW, Gong Y, Padmanabhan S et al. Pharmacogenomic association of nonsynonymous SNPs in SIGLEC12, A1BG, and the selectin region and cardiovascular outcomes. Hypertension 62, 48-54 (2013).

15. O'Donnell PH, Wadhwa N, Danahey K et al. Pharmacogenomics-based point-of-care clinical decision support significantly alters drug prescribing. Clin. Pharmacol. Ther. 102, 859-869 (2017).

16. Rosenman MB, Decker B, Levy KD et al. Lessons learned when introducing pharmacogenomic panel testing into clinical practice. Value Health 20, 54-59 (2017).

17. Kelley EF, Snyder EM, Alkhatib NS et al. Economic evaluation of a pharmacogenomic multi-gene panel test to optimize anti-hypertension therapy: simulation study. J. Med. Econ. 21, 1246-1253 (2018). 